\title{
Nerve Growth Factor Promotes Lysyl Oxidase- dependent Chondrosarcoma Cell Metastasis by Suppressing miR-149-5p Synthesis
}

Huey-En Tzeng

Taipei Medical University

Syuan-Ling Lin

China Medical University Hospital

Louis Anoop Thadevoos

China Medical University

Ming-Yu Lien

China Medical University Hospital

Wei-Hung Yang

Taichung Hospital

Chih-Yuan Ko

China Medical University Hospital

Chih-Yang Lin

China Medical University

Yu-Wen Huang

China Medical University

Ju-Fang Liu

Taipei Medical University

Yi-Chin Fong

China Medical University Beigang Hospital

Hsien-Te Chen

China Medical University Hospital

Chih-Hsin Tang ( $\nabla$ chtang@mail.cmu.edu.tw)

China Medical University https://orcid.org/0000-0002-7113-8352

\section{Research}

Keywords: Nerve growth factor, Chondrosarcoma, Metastasis, Lysyl oxidase, miR-149-5p

Posted Date: July 19th, 2021

DOl: https://doi.org/10.21203/rs.3.rs-704561/v1 
License: (c) (i) This work is licensed under a Creative Commons Attribution 4.0 International License. Read Full License

Version of Record: A version of this preprint was published at Cell Death \& Disease on November 23rd, 2021. See the published version at https://doi.org/10.1038/s41419-021-04392-2. 


\section{Abstract}

Background: The effects of Nerve growth factor (NGF) in chondrosarcoma are not confirmed, although NGF is capable of promoting the progression and metastasis of several different types of tumors. Here we aim to explore the role of NGF in chondrosarcoma and elucidate how NGF acts.

Methods: Immunohistochemistry (IHC)-stained tissue samples from chondrosarcoma patients were stained with NGF and LOX antibodies. Cell migration was examined by Transwell migration and invasion assays. The expression levels of LOX, microRNA-149-5p (miR-149-5p) were measured by quantitative realtime polymerase chain reaction. LOX, PI3K, Akt, and mTOR protein expression were examined by Western blot assays. The interaction between LOX 3'-UTRs and miR-149-5p binding site was explored by luciferase assay. We established the orthotopic in vivo model of chondrosarcoma lung metastasis to further investigate the promoting effects of NGF in metastatic chondrosarcoma.

Results: Here, we found that the levels of NGF and lysyl oxidase (LOX) correlated with tumor stage in patients with chondrosarcoma. NGF facilitated LOX-dependent cellular migration in human chondrosarcoma JJ012 cells, while overexpression of NGF enhanced lung metastasis in a mouse model of chondrosarcoma. NGF promoted LOX synthesis and cell migration by inhibiting miR-149-5p expression through the PI3K, Akt and mTOR signaling cascades. NGF appears to be a worthwhile therapeutic target in the treatment of metastatic chondrosarcoma.

Conclusions: Our study has identified that NGF promotes LOX-dependent cell migration in human chondrosarcoma tissue by inhibiting miR-149-5p synthesis via the PI3K, Akt and mTOR signaling cascades

\section{Background}

Chondrosarcoma is a common malignancy that occurs typically in cartilage-enriched bone (e.g., femur, tibia, or pelvis) $(1,2)$ that displays a high propensity to metastasize to distant organs (1). High-grade chondrosarcomas are particularly prone to metastasize to the lungs $(3,4)$, so delaying or inhibiting this phenomenon is essential for patient survival. Metastasis of any tumor is characterized by the secretion of proteolytic enzymes such as matrix metalloproteinases (MMPs) and lysyl oxidase (LOX), capable of degrading the extracellular matrix (ECM) and basement membrane $(5,6)$. LOX is an extracellular cuproenzyme necessary for the catalyzation of collagen and elastin crosslinking in the ECM (7). Several different tumors, such as oral, breast and gastric cancers, overexpress LOX $(8,9)$, while increased LOX expression in premalignant host tissue is associated with a higher tumor incidence and burden (10). Moreover, the alteration of the ECM by LOX-induced catalyzing of collagen and elastin crosslinkages is thought to be one way in which LOX promotes metastasis: this alteration of the ECM helps to form microenvironments that recruit nonmalignant host cells into the primary tumor microenvironment, and these alterations to the local microenvironments enable premetastatic "permissive niches" to emerge that 
allow colonization of tumor cells and formation of secondary metastases (11). Thus, inhibiting LOX expression would appear to be a useful therapeutic tactic in tumor metastasis.

MicroRNAs (miRNAs) are involved in the cellular processes of many human diseases, including cancers, by regulating different activities of the tumor cell, including apoptosis, proliferation, angiogenesis, drug resistance and metastasis (12). The importance of miRNAs in tumorigenesis is underlined by the fact that they target key metabolic enzymes and protein messenger RNAs (mRNAs) (13). In particular, researchers have suggested that miRNA mimics or inhibitors of metabolic processes and gene regulatory events could improve overall survival in lung cancer (13). Moreover, miRNA levels may serve as potential biomarkers and therapeutic targets in cancers (14). Notably, miRNA levels of LOX are regulated by miR149 (15), while miR-29 contributes to the maintenance of ECM homeostasis by adversely regulating ECM protein production $(15,16)$.

Nerve growth factor (NGF) is vital for neuronal cell growth, apoptosis and differentiation (17). The binding of NGF to its receptor tropomyosin receptor kinase A (TrkA) activates intracellular signaling, immune cell proliferation, differentiation and survival (18). It has also been suggested that NGF plays an integral part in the progression of several types of malignancies, such as ovarian, prostate, and liver cancers (19-21), as well as metastasis in various tumors (21-23), although the role of NGF in chondrosarcoma is uncertain. We therefore set out to define how NGF could affect chondrosarcoma metastasis, in in vitro and in vivo explorations. We also sought to determine whether LOX mediates NGFinduced migration and invasion abilities of chondrosarcoma cells, and whether this process is regulated by PI3K, Akt and mTOR signaling. Finally, we examined potential candidate miRNAs that could affect LOX mRNA expression in chondrosarcoma cell lines, and the potential role of NGF in this process.

\section{Materials And Methods}

\section{Materials}

NGF, LOX, p85, Akt, mTOR and $\beta$-actin antibodies were purchased from GeneTex International Corporation (Hsinchu City, Taiwan). The phosphorylated forms of p85, Akt and mTOR antibodies were purchased from Cell Signaling Technology (Danvers, MA, USA). All ON-TARGET plus short interfering (si)RNAs were obtained from Dharmacon (Lafayette, CO, USA). Quantitative polymerase chain reaction (qPCR) primers and probes, as well as Taqman ${ }^{\circledR}$ One-Step PCR Master Mix, were supplied by Applied Biosystems (Foster City, CA, USA). Recombinant human NGF was obtained from PerpoTech (Rocky Hill, NJ, USA). All other chemicals used in this study were supplied by Sigma-Aldrich (St. Louis, MO, USA).

\section{Cell culture}

The human chondrosarcoma cell line JJ012 was kindly provided by Dr. Sean P. Scully (University of Miami School of Medicine, Miami, FL, USA), while the SW1353 chondrosarcoma cell line was obtained from the American Type Cell Culture Collection (Manassas, VA, USA). JJ012 cells stably expressing the 
NGF complementary DNA (cDNA) clone (JJ012/NGF cells) were established according to our previous method (24). Cells were cultured 50\%/50\% in Dulbecco's Modified Eagle Medium (DMEM)/alphaminimum essential medium (a-MEM) medium, 10\% fetal bovine serum (FBS) and antibiotics, then maintained in a humidified incubator at $37^{\circ} \mathrm{C}$ in $5 \% \mathrm{CO}_{2}$.

\section{Cell migration and invasion assay}

Chondrosarcoma cells were seeded into the upper chamber of Transwell plates (Costar, NY, USA) precoated with a layer of Matrigel for the invasion assay. NGF and pharmaceutical inhibitors were added to the lower chamber. After $18 \mathrm{~h}$ of incubation, migrated cells were fixed with $3.7 \%$ formaldehyde and stained with crystal violet, then counted manually under the microscope $(25,26)$.

\section{Western blot analysis}

After the indicated treatments, chondrosarcoma cells were lysed in RIPA buffer. The extracted proteins were resolved by SDS-PAGE and transferred to Immobilon ${ }^{\circledR}$ polyvinylidene fluoride (PVDF) membranes. Western blot analysis was performed using the methodology described in our previous reports (27-29).

\section{mRNA and miRNA quantification}

Total RNA was extracted from chondrosarcoma cells using TRIzol reagent and RNA concentrations were determined using a NanoVue Plus spectrophotometer (GE Healthcare Life Sciences; Pittsburgh, PA, USA). The M-MLV RT kit (Thermo Fisher Scientific; Waltham, MA, USA) and the Mir- $X^{\text {TM }}$ miRNA First-Strand Synthesis kit (Clontech; Mountain View, CA, USA) were used to perform reverse transcription of total RNA into cDNA. Quantitative real-time PCR (qPCR) analysis was performed according to our previous reports $(30,31)$.

\section{Luciferase assay}

The human LOX luciferase reporter plasmids containing wild-type or mutant sequences of the three prime untranslated region (3'-UTR) encompassing miR-149-5p binding sites obtained from MDBio Inc. (Taipei, Taiwan). Chondrosarcoma cells were transfected with the plasmids using Lipofectamine 2000 (Thermo Fisher Scientific; Waltham, MA, USA), then stimulated with NGF for 24 h. Luciferase activity was monitored using a luciferase assay kit $(30,32,33)$.

\section{Tumor xenograft study}


Four-week-old male BALB/c nude mice (8 in each group) were bought from Taipei's National Laboratory Animal Center and orthotopically injected with JJ012 or JJ012/NGF cells $\left(5 \times 10^{6}\right.$, resuspended in $100 \mu \mathrm{L}$ of medium containing $50 \%$ serum-free DMEM/a-MEM and $50 \%$ Matrigel), according to a previous protocol (24). Tumor growth in the tibiae was monitored each week by bioluminescence imaging using a Xenogen IVIS imaging system 200 (PerkinElmer, MA, USA). At 12 weeks, the mice were euthanized by $\mathrm{CO}_{2}$ inhalation. The lungs were removed and fixed in $10 \%$ formalin for further analysis. All animal procedures were approved and performed in accordance with the guidelines of the Institutional Animal Care and Use Committee of China Medical University (CMUIACUC-2019-079).

\section{Collection of clinical patient samples}

Human cartilage specimens and tumor specimens were obtained during primary total knee arthroplasty and from patients diagnosed with chondrosarcoma undergoing orthopedic surgery at China Medical University Hospital, with approval granted by the local Institutional Review Board. All study participants gave written consent before enrollment.

\section{Immunohistochemistry (IHC) staining}

Mouse lung tissues and human specimens were rehydrated and incubated with primary anti-NGF or LOX antibodies, then treated with biotin-labeled secondary antibody. Finally, the slides were examined using the ABC Kit (Vector Laboratories, CA, USA) and photographed using the microscope.

\section{Statistics}

All values are presented as the mean \pm standard deviation (SD). Significance testing on the difference between the groups was assessed by the Student's $t$-test and considered significant if the $p$-value was $<0.05$.

\section{Results}

\section{NGF promotes LOX-2-dependent migration and invasion of chondrosarcoma}

NGF is associated with cancer cell progression and survival in several different types of cancers $(34,35)$. We first investigated the effects of NGF upon cell motility in chondrosarcoma cell lines JJ012 and SW1353. Treatment of chondrosarcoma cells with NGF promoted migration and invasion ability, according to Transwell assay data (Fig. 1A-D). LOX reportedly mediates cell migration and metastasis of different cancers (11). We therefore examined whether LOX plays a role in NGF-induced migration and invasion in chondrosarcoma. Stimulation of cells with NGF enhanced LOX mRNA and protein expression 
(Fig. 1E\&F). Transfection of cells with LOX siRNA antagonized NGF-induced promotion of migration and invasion (Fig. 1G-I), implying that LOX is critical to NGF-promoted chondrosarcoma cell migration and invasion.

\section{PI3K, Akt and mTOR signaling regulates NGF-induced promotion of LOX-mediated chondrosarcoma cell migration and invasion}

PI3K and Akt signaling helps to drive chondrosarcoma metastasis $(24,36)$. Treating cells with PI3K inhibitors (Ly294002 and wortmannin) or an Akt inhibitor (Akt i) significantly reduced NGF-enhanced promotion of cell motility and LOX synthesis (Fig. 2A-F and 3A-F). Similar effects were observed when the chondrosarcoma cells were transfected with p85 or Akt siRNAs (Fig. 2B-G and 3B-G). NGF stimulation time-dependently induced p85 and Akt phosphorylation in both cell lines (Fig. 2H \& 3H). PI3K inhibitor treatment antagonized NGF-facilitated Akt phosphorylation (Fig. 3I), indicating that the PI3K/Akt signaling pathway controls NGF-enhanced LOX synthesis, as well as chondrosarcoma cell migration and invasion.

The PI3K/Akt signaling pathway regulates downstream mTOR activity and together they control the metastatic potential of chondrosarcoma (24). Incubation of cells with the mTOR inhibitor rapamycin reduced the effects of NGF upon cell migration and invasion, as well as LOX synthesis (Fig. 4A-E). Transfection of the cells with an mTOR siRNA confirmed the similar effects of NGF (Fig. 4B-G). Treatment of chondrosarcoma cell lines with NGF upregulated mTOR phosphorylation (Fig. 4H), while PI3K and Akt inhibitors reversed NGF-mediated mTOR phosphorylation (Fig. 4I), suggesting that PI3/Akt-dependent mTOR activation mediates NGF-promoted increases in LOX expression and cell motility.

\section{The miR-149-5p/LOX axis regulates NGF-enhanced stimulation of chondrosarcoma cell migration and invasion}

miRNA-associated regulation of LOX expression is a critical mechanism in the progression and metastasis of cancer cells $(16,37)$. A search of five online databases for miRNA target prediction indicated that the 3'-UTR region of LOX mRNA contains 14 promising candidate miRNAs (Fig. 5A\&B). Treatment of JJ012 cells with NGF ( $100 \mathrm{ng} / \mathrm{mL}$ ) significantly diminished miR-149-5p expression (Fig. 5B) and at the concentrations of 30,50 , or $100 \mathrm{ng} / \mathrm{mL}$, obviously inhibited miR-149-5p synthesis in both chondrosarcoma cell lines, in a concentration-dependent manner (Fig. 5C). Transfection of cells with miR149-5p mimic significantly reduced NGF-induced stimulation of cell migration and LOX mRNA expression in both chondrosarcoma cell lines (Fig. 5D-F). Next, we investigated whether PI3K, Akt and mTOR signaling regulate NGF-induced suppression of miR-149-5p synthesis. PI3K, Akt and mTOR inhibitors, and their respective siRNAs, all reversed NGF-induced inhibition of miR-149-5p expression (Fig. 5G\&H). Analyses of the LOX 3'-UTR luciferase plasmids revealed that NGF increased luciferase activity of the 


\section{Overexpression of NGF promotes chondrosarcoma metastasis to lungs in mice}

We used the orthotopic in vivo model of chondrosarcoma lung metastasis to examine the stimulatory effects of NGF in metastatic chondrosarcoma (24). JJ012/NGF cells expressed high protein levels of both NGF and LOX, and they exhibited a high migratory ability (Fig. 6A-C). JJ012 and JJ012/NGF cells were orthotopically implanted into the right leg tibia and tumor size was monitored by the IVIS system (Fig. 6D\&E). Overexpression of NGF significantly increased tumor growth in the tibia (Fig. 6D\&E). At 12 weeks, metastasis to the lung was significantly more likely with JJ012/NGF cells than with JJ012 cells (Fig. 6F\&G). IHC results revealed significant increases in the levels of NGF and LOX expression in the JJ012/NGF orthotopic model (Fig. 6H), confirming that NGF facilitates the metastasis of chondrosarcoma to the mouse lung.

\section{NGF and LOX levels are positively correlated in human chondrosarcoma tissue}

Finally, we investigated NGF and LOX expression in patients with chondrosarcoma. IHC results revealed higher levels of NGF and LOX expression in patients with higher grade chondrosarcoma than in those with lower grade disease; the levels of NGF and LOX expression were reflected by tumor stage (Fig. 7A-C). These results are quantified in Figures 7D\&E, which illustrate how the levels of NGF and LOX expression were significantly higher in higher-stage tumors (IIA and IIB) than in lower-stage tumors (IA and IB). A positive correlation observed between NGF and LOX staining intensity of human chondrosarcoma tissue $\left(r^{2}=0.534\right.$, Fig. $\left.7 F\right)$ indicates that the levels of these proteins are associated with the progression of chondrosarcoma disease.

\section{Discussion}

Chondrosarcoma is a malignant bone neoplasm that constitutes almost one-third ( $26 \%)$ of all bone cancers (38). Chemotherapy and radiotherapy have very limited effectiveness, so surgery is the major therapeutic modality for chondrosarcoma. This malignancy is notorious for its aggressive clinical course and propensity to metastasize (39). An effective adjuvant therapy is urgently needed to suppress chondrosarcoma metastasis $(1,3)$. NGF plays an important role in tumor cell proliferation, migration and survival $(34,35)$. Our investigation has found that levels of NGF and LOX expression are positively correlated with tumor staging in patients with chondrosarcoma. We also confirmed that NGF facilitates LOX-dependent chondrosarcoma cell migration and metastasis by suppressing miR-149-5p synthesis via $\mathrm{PI} 3 \mathrm{~K}$, Akt and mTOR signaling. 
The localization of LOX to the ECM, especially in cancer cells, makes it an attractive target for activated prodrugs that tend to accumulate in the tumor stroma (7). The crosslinking of collagen and elastin facilitated by LOX enhances the proliferation of tumor cells and promotes metastatic colonization and a fibrotic microenvironment that improves the survival of tumor cells, supporting the development of metastasis $(11,40)$. Therapy that targets LOX lessens the severity of fibrosis and also restricts metastatic colonization boosted by fibrosis (40). Our study is the first to describe an association between levels of LOX expression and tumor stage in chondrosarcoma tissue specimens. Our in vitro and in vivo evidence suggests that NGF facilitates LOX-dependent chondrosarcoma metastasis. LOX is therefore a promising molecular targeting for treating chondrosarcoma metastasis.

Activation of the PI3K/Akt pathway is important for regulating various cellular functions (41). This signaling pathway also regulates the metastatic potential of human chondrosarcoma (24). Our study results show that NGF promotes phosphorylation of PI3K and Akt, while PI3K and Akt pharmacological inhibitors suppress NGF-induced promotion of LOX expression, chondrosarcoma cell migration and invasion. This phenomenon was confirmed by similar effects observed with genetic siRNAs of PI3K and Akt. Previous evidence stating that the PI3K/Akt pathway is an upstream molecule of mTOR, with the capacity to regulate cell motility (42), was confirmed by our data showing that both an mTOR inhibitor and siRNA antagonized NGF-induced LOX production and cell motility. PI3K and Akt inhibitors also inhibited NGF-promoted phosphorylation of mTOR, indicating that PI3K/Akt-dependent mTOR activation mediates NGF-facilitated promotion of LOX synthesis and chondrosarcoma cell motility.

MiRNAs post-transcriptionally regulate gene expression (43). During tumor metastasis, aberrant miRNA expression mediates cancer cell migration and invasion (44). Our analysis of five open-source databases identified that 14 miRNAs potentially interfere with LOX transcription. NGF significantly lowered miR-149$5 p$ expression. We enhanced miR-149-5p levels in chondrosarcoma cells by transfecting them with a specific miR-423-5p mimic, which markedly reduced LOX synthesis and the migratory capacity of the cells. miR-423-5p expression was negatively correlated with LOX expression, as well as the migratory and invasive activities of chondrosarcoma cells. Thus, our evidence has identified that miR-149-5p exhibits novel antimetastatic properties.

\section{Conclusion}

Our study has identified that NGF promotes LOX-dependent migratory and invasive activities in human chondrosarcoma cells by suppressing miR-149-5p synthesis via the PI3K, Akt and mTOR signaling cascades (Fig. 8). It appears to be worth targeting NGF expression in metastatic chondrosarcoma.

\section{Abbreviations}

NGF, Nerve growth factor; LOX, lysyl oxidase; MMPs, matrix metalloproteinases; ECM, extracellular matrix; miRNAs, MicroRNAs; mRNAs, messenger RNAs; TrkA, tropomyosin receptor kinase A; qPCR, Quantitative polymerase chain reaction; cDNA, complementary DNA; DMEM, Dulbecco's Modified Eagle Medium; $a-$ 
MEM, alpha-minimum essential medium; FBS, fetal bovine serum; PVDF, polyvinylidene fluoride; 3'-UTR, three prime untranslated region; IHC, Immunohistochemistry.

\section{Declarations}

\section{Ethics approval and consent to participate}

The authors declare that the data supporting the findings of this study are available within the article. All animal procedures were approved and performed in accordance with the guidelines of the Institutional Animal Care and Use Committee of China Medical University (CMUIACUC-2019-079).

\section{Consent for publication}

Not applicable.

\section{Availability of data and materials}

The datasets used and/or analyzed during the present study are available from the corresponding author for reasonable request.

\section{Competing interests}

The authors declare that they have no competing interests.

\section{Funding}

This work was supported by grants from the Ministry of Science and Technology of Taiwan (MOST 1092320-B-039-065, MOST 108-2314-B-039-034-MY3) and China Medical University Hospital, Taichung, Taiwan (DMR-109-013, DMR-109-236, DMR-110-105, DMR-110-017, DMR-110-180).

\section{Authors' contributions}

HET, SLL, and CHT conceived and designed the experiments. LAT, MYL, WHY, and CYK performed the experiments. HET, SLL, and LAT contributed to perform in vitro and in vivo experiments. CYL, YWH, and JFL contributed to analyze experiment data. YCF, HTC, and CHT contributed to the writing of the manuscript. All authors approved for publication.

\section{Acknowledgments}


We would like to thank lona J. MacDonald from China Medical University for her English language revision of this manuscript.

\section{Author details}

${ }^{1}$ Ph.D. Program for Cancer Molecular Biology and Drug Discovery, College of Medical Science and Technology, Taipei Medical University, Taipei, Taiwan. ${ }^{2}$ Graduate Institute of Cancer Biology and Drug Discovery, College of Medical Science and Technology, Taipei Medical University, Taipei, Taiwan.

${ }^{3}$ Division of Hematology/Oncology, Department of Medicine, Taipei Medical University Hospital, Taiwan. ${ }^{4}$ School of Medicine, College of Medicine, Taipei Medical University, Taipei, Taiwan. ${ }^{5}$ Translational Medicine Research Center, China Medical University Hospital, Taichung, Taiwan. ${ }^{6}$ International Master Program of Biomedical Sciences, China Medical University, Taichung, Taiwan. ${ }^{7}$ Division of Hematology and Oncology, Department of Internal Medicine, China Medical University Hospital, Taichung, Taiwan. ${ }^{8}$ Graduate Institute of Basic Medical Science, China Medical University, Taichung, Taiwan. ${ }^{9}$ School of Chinese Medicine, China Medical University, Taichung, Taiwan. ${ }^{10}$ Department of Nursing, National Taichung University of Science and Technology, Taichung, Taiwan. ${ }^{11}$ Department of Orthopedic Surgery, Taichung Hospital, Ministry of Health and Welfare, Taichung, Taiwan. ${ }^{12}$ Department of Orthopedic Surgery, China Medical University Hospital, Taichung, Taiwan. ${ }^{13}$ Department of Pharmacology, School of Medicine, China Medical University, Taichung, Taiwan. ${ }^{14}$ Graduate Institute of Biomedical Sciences, China Medical University, Taichung, Taiwan. ${ }^{15}$ School of Oral Hygiene, College of Oral Medicine, Taipei Medical University, Taipei, Taiwan. ${ }^{16}$ Department of Sports Medicine, College of Health Care, China Medical University, Taichung, Taiwan. ${ }^{17}$ Department of Orthopedic Surgery, China Medical University Beigang Hospital, Yunlin, Taiwan. ${ }^{18}$ Department of Biotechnology, College of Health Science, Asia University, Taichung, Taiwan. ${ }^{19}$ Chinese Medicine Research Center, China Medical University, Taichung, Taiwan

\section{References}

1. MacDonald IJ, Lin CY, Kuo SJ, Su CM, Tang CH. An update on current and future treatment options for chondrosarcoma. Expert Rev Anticancer Ther. 2019;19(9):773-86.

2. Chen PC, Cheng HC, Yang SF, Lin CW, Tang CH. The CCN family proteins: modulators of bone development and novel targets in bone-associated tumors. BioMed research international. 2014;2014:437096.

3. Chen JC, Fong YC, Tang $\mathrm{CH}$. Novel strategies for the treatment of chondrosarcomas: targeting integrins. BioMed research international. 2013;2013:396839.

4. Ferguson JL, Turner SP. Bone Cancer: Diagnosis and Treatment Principles. Am Family Phys. 2018;98(4):205-13.

5. Fan TM, Roberts RD, Lizardo MM. Understanding and Modeling Metastasis Biology to Improve Therapeutic Strategies for Combating Osteosarcoma Progression. Frontiers in oncology. 2020;10:13. 
6. Steeg PS. Targeting metastasis. Nature reviews Cancer. 2016;16(4):201-18.

7. Granchi C, Funaioli T, Erler JT, Giaccia AJ, Macchia M, Minutolo F. Bioreductively activated lysyl oxidase inhibitors against hypoxic tumours. ChemMedChem. 2009;4(10):1590-4.

8. Osawa T, Ohga N, Akiyama K, Hida Y, Kitayama K, Kawamoto T, et al. Lysyl oxidase secreted by tumour endothelial cells promotes angiogenesis and metastasis. British journal of cancer. 2013;109(8):2237-47.

9. Kirschmann DA, Seftor EA, Fong SF, Nieva DR, Sullivan CM, Edwards EM, et al. A molecular role for lysyl oxidase in breast cancer invasion. Cancer research. 2002;62(15):4478-83.

10. Grau-Bove X, Ruiz-Trillo I, Rodriguez-Pascual F. Origin and evolution of lysyl oxidases. Scientific reports. 2015;5:10568.

11. Johnston KA, Lopez KM. Lysyl oxidase in cancer inhibition and metastasis. Cancer letters. 2018;417:174-81.

12. Tutar Y, Ozgur A, Tutar E, Tutar L, Pulliero A, Izzotti A. Regulation of oncogenic genes by MicroRNAs and pseudogenes in human lung cancer. Biomedicine pharmacotherapy = Biomedecine pharmacotherapie. 2016;83:1182-90.

13. Iqbal MA, Arora S, Prakasam G, Calin GA, Syed MA. MicroRNA in lung cancer: role, mechanisms, pathways and therapeutic relevance. Mol Aspects Med. 2019;70:3-20.

14. Mohammadi A, Mansoori B, Baradaran B. The role of microRNAs in colorectal cancer. Biomedicine pharmacotherapy $=$ Biomedecine pharmacotherapie. 2016;84:705-13.

15. Liu H, Brannon AR, Reddy AR, Alexe G, Seiler MW, Arreola A, et al. Identifying mRNA targets of microRNA dysregulated in cancer: with application to clear cell Renal Cell Carcinoma. BMC systems biology. 2010;4:51.

16. Yin H, Wang Y, Wu Y, Zhang X, Zhang X, Liu J, et al. EZH2-mediated Epigenetic Silencing of miR29/miR-30 targets LOXL4 and contributes to Tumorigenesis, Metastasis, and Immune Microenvironment Remodeling in Breast Cancer. Theranostics. 2020;10(19):8494-512.

17. Lewin GR, Barde YA. Physiology of the neurotrophins. Annu Rev Neurosci. 1996;19:289-317.

18. Minnone G, De Benedetti F, Bracci-Laudiero L. NGF and Its Receptors in the Regulation of Inflammatory Response. International journal of molecular sciences. 2017;18(5).

19. Garrido MP, Torres I, Avila A, Chnaiderman J, Valenzuela-Valderrama M, Aramburo J, et al. NGF/TRKA Decrease miR-145-5p Levels in Epithelial Ovarian Cancer Cells. International journal of molecular sciences. 2020;21(20).

20. Chen WY, Wen YC, Lin SR, Yeh HL, Jiang KC, Chen WH, et al. Nerve growth factor interacts with CHRM4 and promotes neuroendocrine differentiation of prostate cancer and castration resistance. Communications biology. 2021;4(1):22.

21. Lin $\mathrm{H}$, Huang $\mathrm{H}, \mathrm{Yu} Y$, Chen $W$, Zhang $\mathrm{S}$, Zhang Y. Nerve growth factor regulates liver cancer cell polarity and motility. Molecular medicine reports. 2021;23(4). 
22. Singh R, Karri D, Shen H, Shao J, Dasgupta S, Huang S, et al. TRAF4-mediated ubiquitination of NGF receptor TrkA regulates prostate cancer metastasis. J Clin Investig. 2018;128(7):3129-43.

23. McCaffrey G, Thompson ML, Majuta L, Fealk MN, Chartier S, Longo G, et al. NGF blockade at early times during bone cancer development attenuates bone destruction and increases limb use. Cancer research. 2014;74(23):7014-23.

24. Tzeng HE, Tang CH, Wu SH, Chen HT, Fong YC, Lu YC, et al. CCN6-mediated MMP-9 activation enhances metastatic potential of human chondrosarcoma. Cell death disease. 2018;9(10):955.

25. Lee HP, Wang SW, Wu YC, Lin LW, Tsai FJ, Yang JS, et al. Soya-cerebroside inhibits VEGF-facilitated angiogenesis in endothelial progenitor cells. Food Agr Immunol. 2020;31(1):193-204.

26. Hou CH, Lin FL, Hou SM, Liu JF. Cyr61 promotes epithelial-mesenchymal transition and tumor metastasis of osteosarcoma by Raf-1/MEK/ERK/Elk-1/TWIST-1 signaling pathway. Mol Cancer. 2014;13:236.

27. Lee HP, Chen PC, Wang SW, Fong YC, Tsai CH, Tsai FJ, et al. Plumbagin suppresses endothelial progenitor cell-related angiogenesis in vitro and in vivo. J Funct Foods. 2019;52:537-44.

28. Lee HP, Wang SW, Wu YC, Tsai CH, Tsai FJ, Chung JG, et al. Glucocerebroside reduces endothelial progenitor cell-induced angiogenesis. Food Agr Immunol. 2019;30(1):1033-45.

29. Liu SC, Tsai CH, Wu TY, Tsai CH, Tsai FJ, Chung JG, et al. Soya-cerebroside reduces IL-1 beta-induced MMP-1 production in chondrocytes and inhibits cartilage degradation: implications for the treatment of osteoarthritis. Food Agr Immunol. 2019;30(1):620-32.

30. Yang YC, Chiou PC, Chen PC, Liu PY, Huang WC, Chao CC, et al. Melatonin reduces lung cancer stemness through inhibiting of PLC, ERK, p38, beta-catenin, and Twist pathways. Environmental toxicology. 2019;34(2):203-9.

31. Wang M, Chao CC, Chen PC, Liu PI, Yang YC, Su CM, et al. Thrombospondin enhances RANKLdependent osteoclastogenesis and facilitates lung cancer bone metastasis. Biochem Pharmacol. 2019;166:23-32.

32. Su CM, Tang CH, Chi MJ, Lin CY, Fong YC, Liu YC, et al. Resistin facilitates VEGF-C-associated lymphangiogenesis by inhibiting miR-186 in human chondrosarcoma cells. Biochem Pharmacol. 2018;154:234-42.

33. Wu TJ, Lin CY, Tsai CH, Huang YL, Tang CH. Glucose suppresses IL-1 beta-induced MMP-1 expression through the FAK, MEK, ERK, and AP-1 signaling pathways. Environmental toxicology. 2018;33(10):1061-8.

34. Aloe L, Rocco ML, Balzamino BO, Micera A. Nerve growth factor: role in growth, differentiation and controlling cancer cell development. Journal of experimental clinical cancer research: CR. 2016;35(1):116.

35. Jiang J, Bai J, Qin T, Wang Z, Han L. NGF from pancreatic stellate cells induces pancreatic cancer proliferation and invasion by PI3K/AKT/GSK signal pathway. J Cell Mol Med. 2020;24(10):5901-10.

36. Lin CY, Chen HJ, Li TM, Fong YC, Liu SC, Chen PC, et al. beta5 integrin up-regulation in brain-derived neurotrophic factor promotes cell motility in human chondrosarcoma. PloS one. 2013;8(7):e67990. 
37. Boufraqech M, Nilubol N, Zhang L, Gara SK, Sadowski SM, Mehta A, et al. miR30a inhibits LOX expression and anaplastic thyroid cancer progression. Cancer research. 2015;75(2):367-77.

38. Leddy LR, Holmes RE. Chondrosarcoma of bone. Cancer treatment research. 2014;162:117-30.

39. Gelderblom H, Hogendoorn PC, Dijkstra SD, van Rijswijk CS, Krol AD, Taminiau AH, et al. The clinical approach towards chondrosarcoma. Oncologist. 2008;13(3):320-9.

40. Cox TR, Bird D, Baker AM, Barker HE, Ho MW, Lang G, et al. LOX-mediated collagen crosslinking is responsible for fibrosis-enhanced metastasis. Cancer research. 2013;73(6):1721-32.

41. Issinger OG, Guerra B. Phytochemicals in cancer and their effect on the PI3K/AKT-mediated cellular signalling. 139: Biomedicine \& pharmacotherapy = Biomedecine \& pharmacotherapie; 2021. p. 111650.

42. Sokolowski KM, Koprowski S, Kunnimalaiyaan S, Balamurugan M, Gamblin TC, Kunnimalaiyaan M. Potential Molecular Targeted Therapeutics: Role of PI3-K/Akt/mTOR Inhibition in Cancer. Anti-cancer Agents Med Chem. 2016;16(1):29-37.

43. Nugent M. MicroRNAs: exploring new horizons in osteoarthritis. Osteoarthritis cartilage. 2016;24(4):573-80.

44. Puppo M, Valluru MK, Clezardin P. MicroRNAs and Their Roles in Breast Cancer Bone Metastasis. Current osteoporosis reports. 2021.

\section{Figures}


(A)

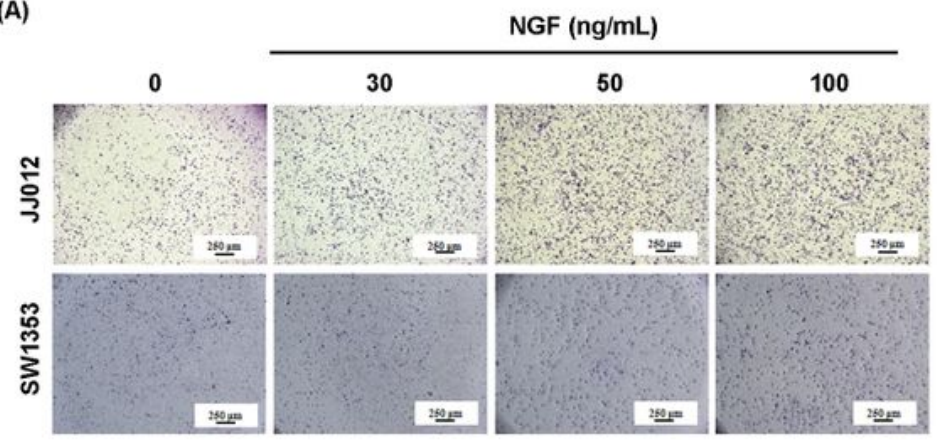

(C)

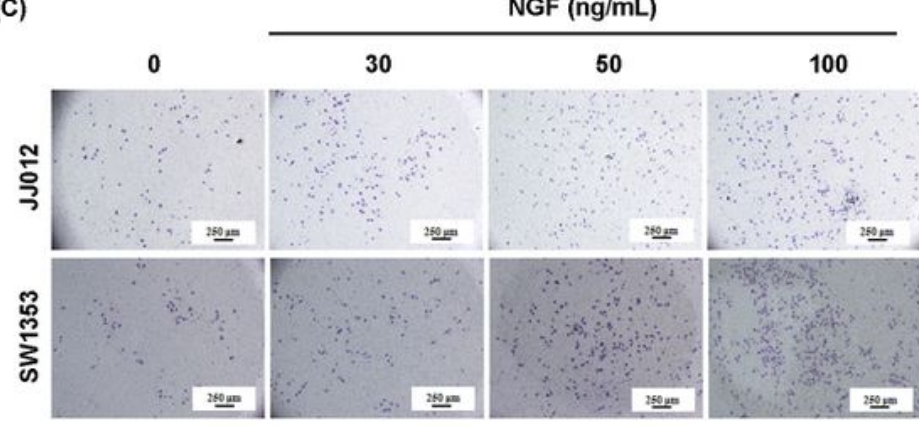

(E)

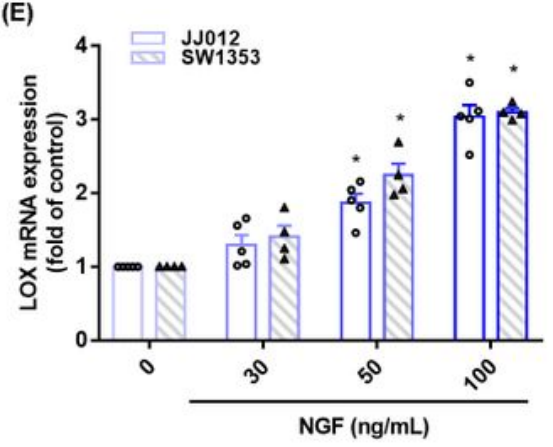

(H)

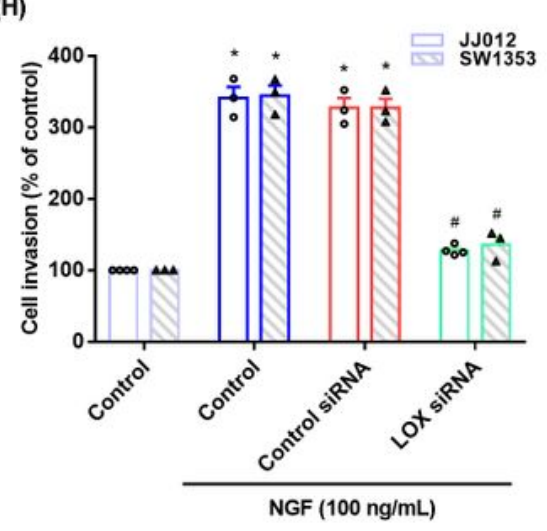

(B)

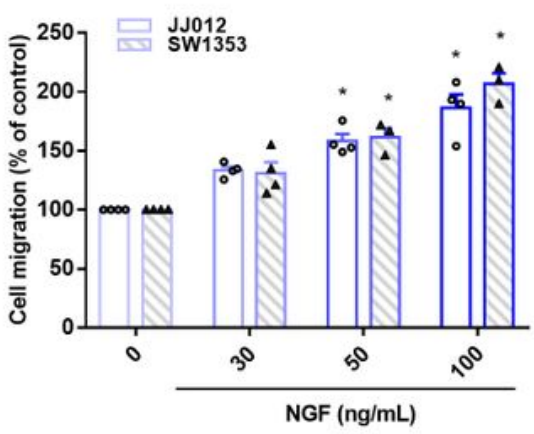

(D)

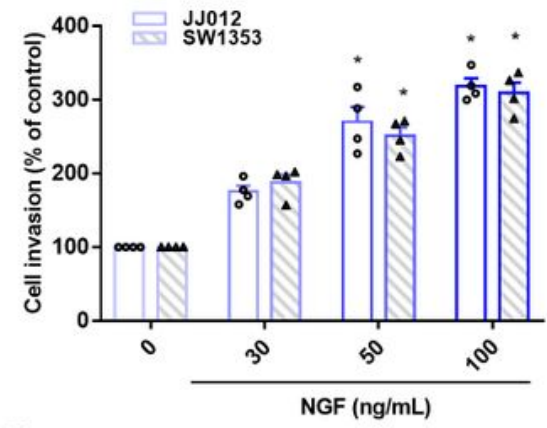

(G)

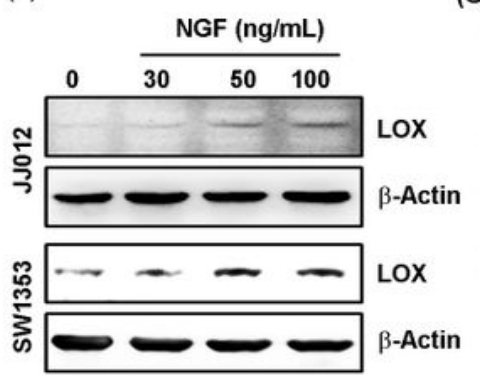

(I)
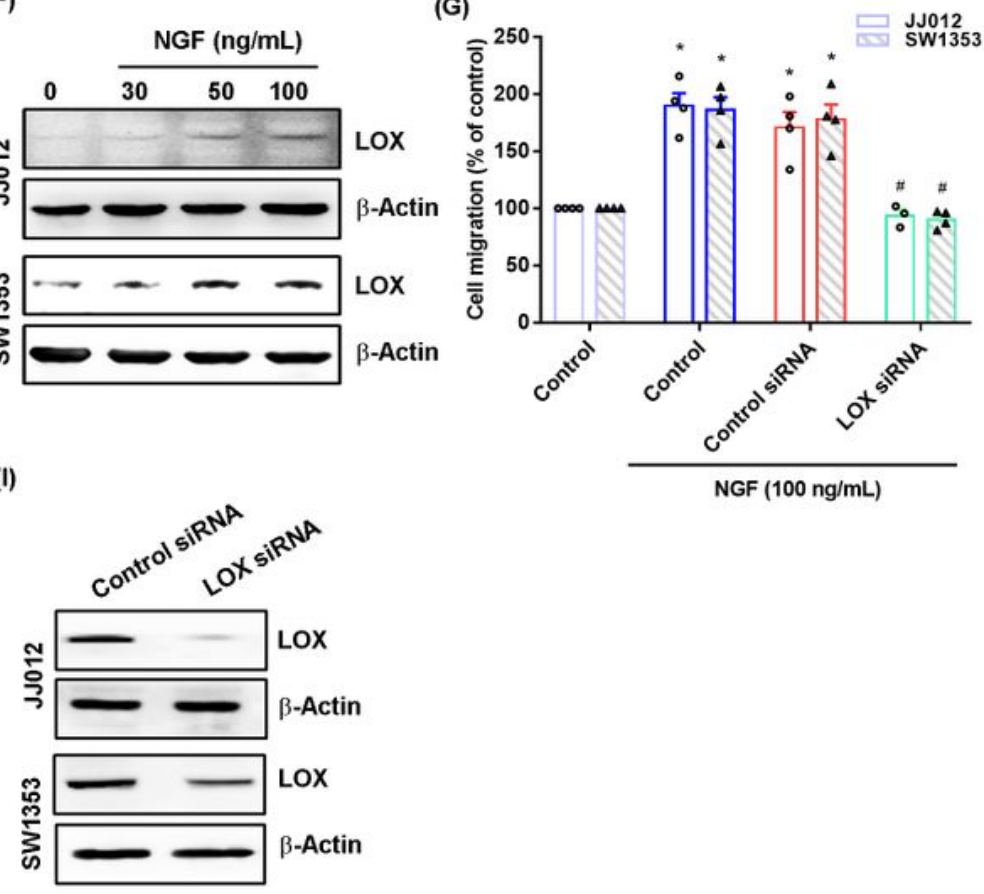

\section{Figure 1}

NGF promotes LOX-dependent cell migration and invasion in human chondrosarcoma. (A-D) Cells were incubated with NGF (30-100 ng/mL) and cell migration and invasion was examined by the Transwell assay. (E-F) Cells were incubated with NGF (30-100 ng/mL) and levels of LOX mRNA and protein expression were examined by qPCR and Western blot assays. (G-I) Cells were transfected with LOX siRNAs, then stimulated with NGF. Cell migration and invasion, as well as levels of LOX expression, were 
examined by Transwell and Western blot assays. * $p<0.05$ compared with the control group; \# $p<0.05$ compared with the NGF-treated group.

(A)

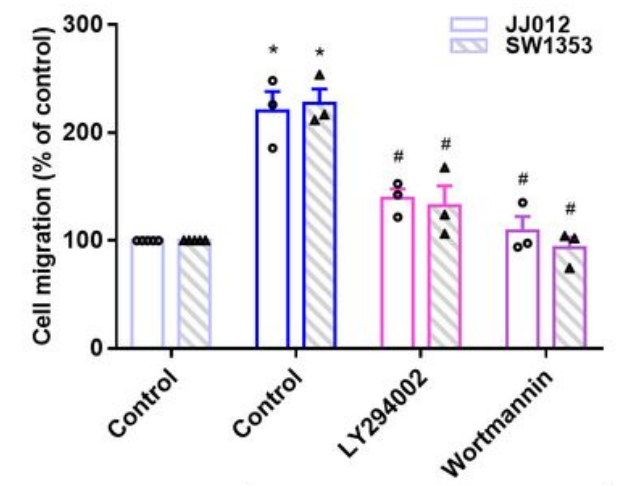

(D)

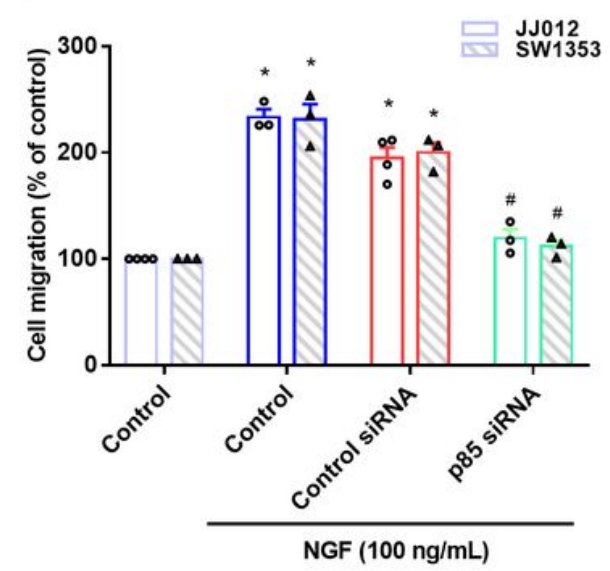

(B)
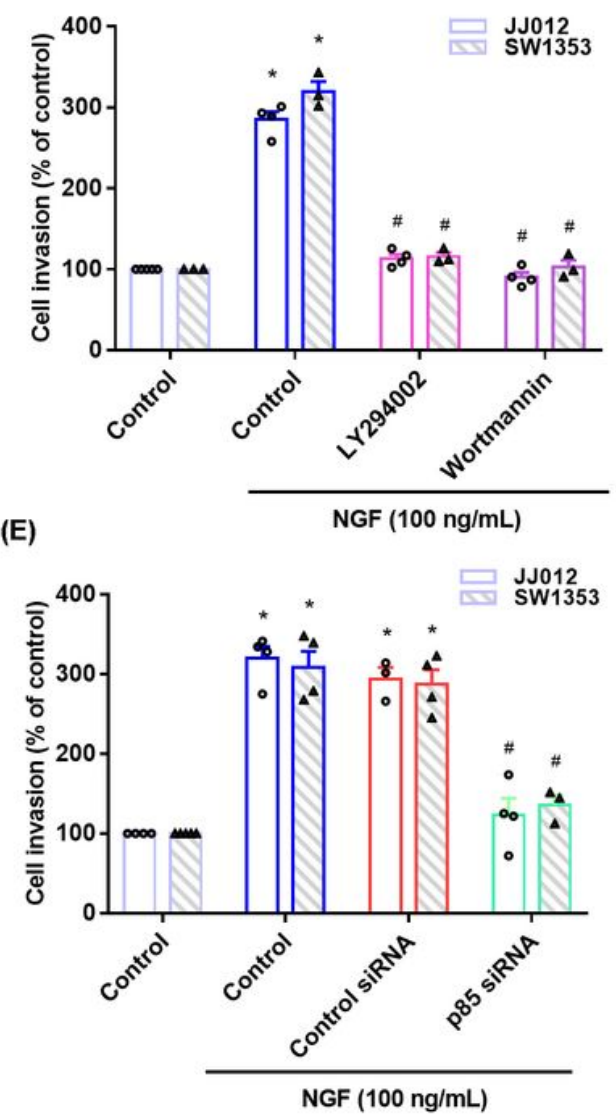

(C)
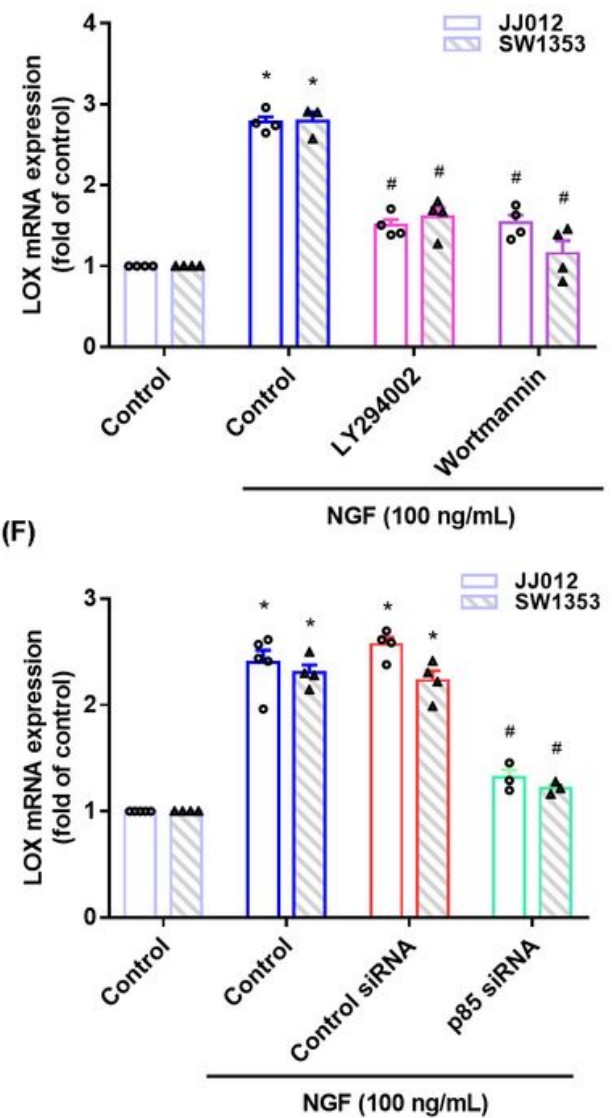

(G)

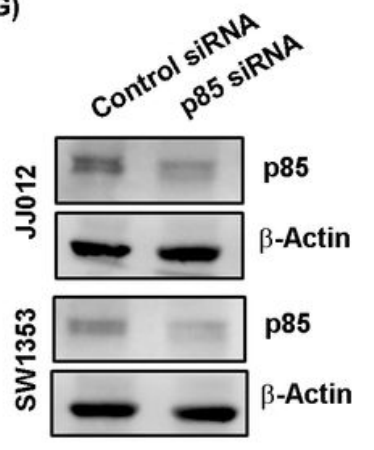

(H)

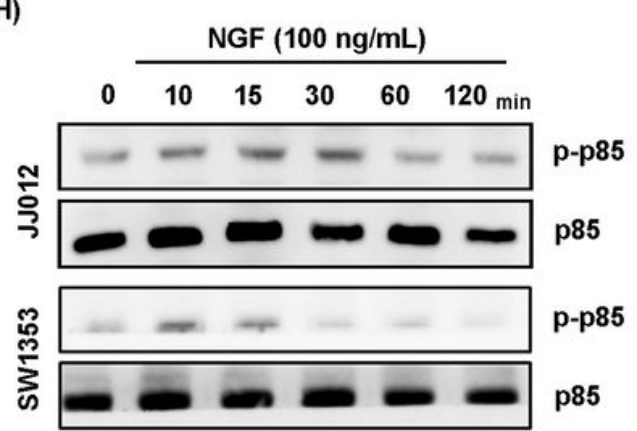

\section{Figure 2}

The PI3K pathway mediates NGF-induced LOX expression and cell motility. (A-F) Cells were treated with PI3K inhibitors (Ly2942002 and wortmannin) or transfected with a p85 siRNA, then stimulated with NGF. Cell migratory and invasive activities, as well as levels of LOX expression, were examined by Transwell and qPCR assays. (G) Cells were transfected with a p85 siRNA and p85 expression was examined by Western blot. $(\mathrm{H})$ Cells were incubated with NGF for the indicated time intervals; p85 phosphorylation was examined by Western blot. * $p<0.05$ compared with the control group; $\# p<0.05$ compared with the NGFtreated group. 
(A)

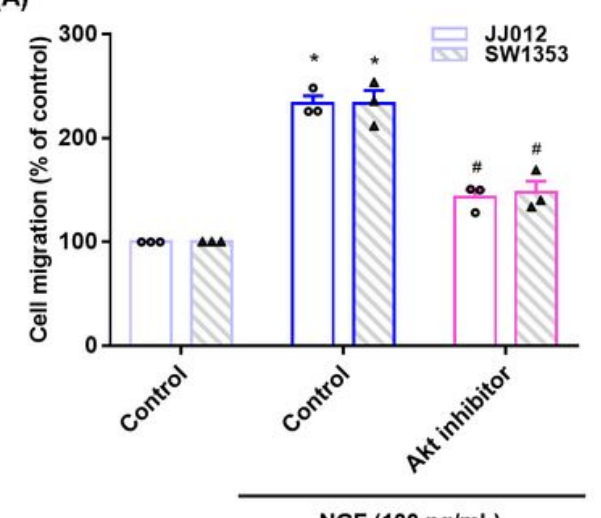

NGF (100 ng/mL)

(D)

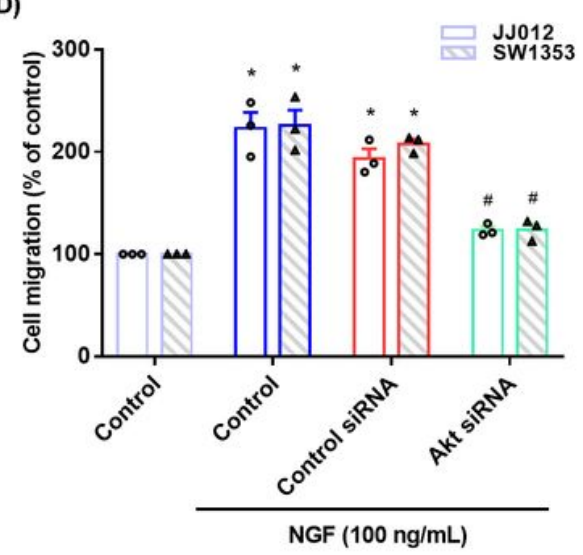

(G)

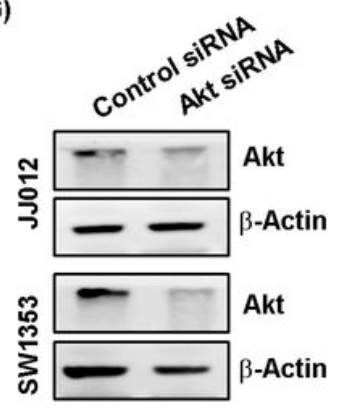

(H)
(B)

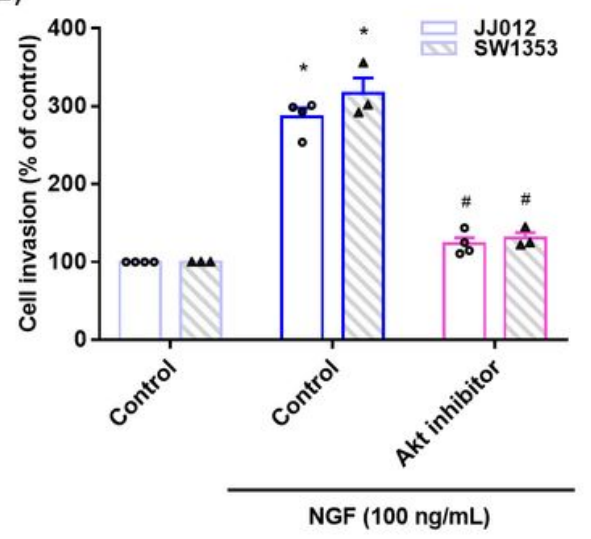

(E)
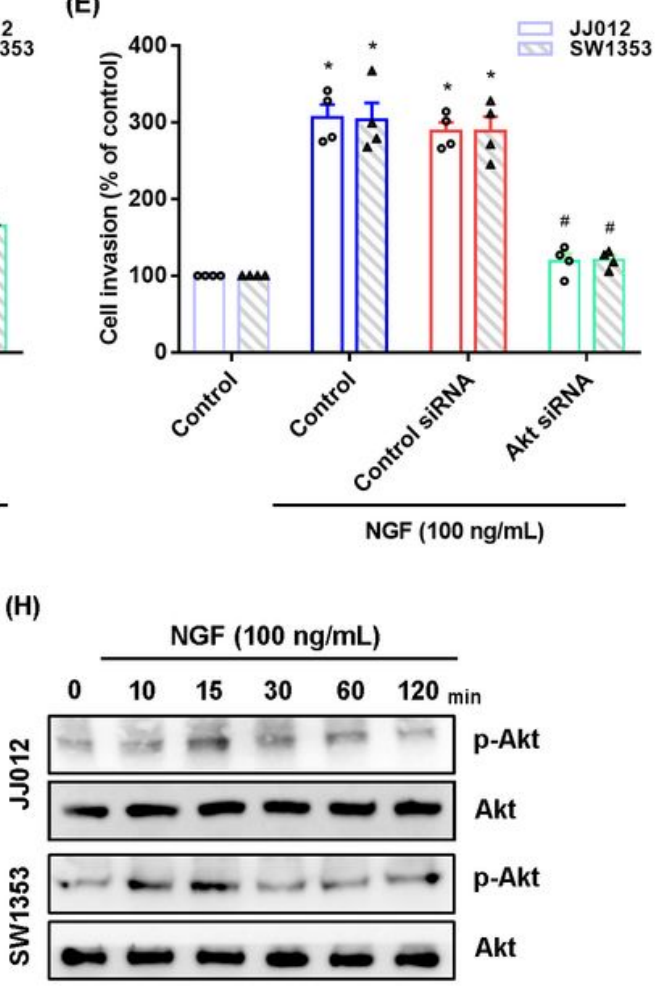

(C)

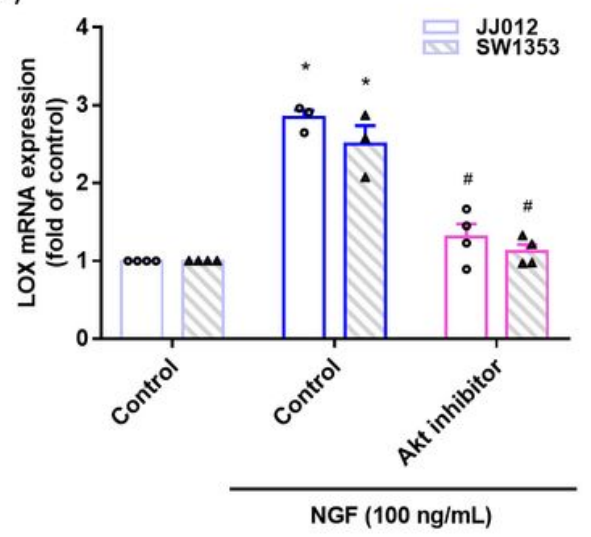

(F)

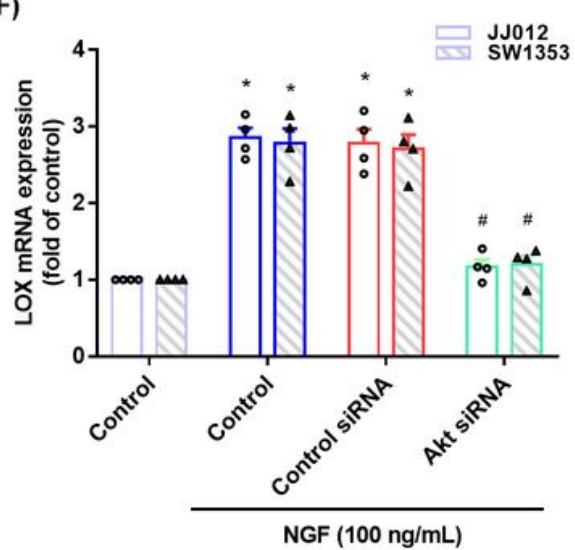

(I)

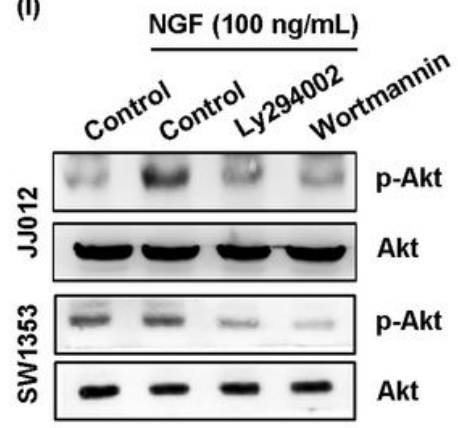

\section{Figure 3}

The Akt pathway mediates NGF-induced LOX expression and cell motility. (A-F) Cells were pretreated with a Akt inhibitor (Akt i) or transfected with a Akt siRNA, then stimulated with NGF. Cell migration and invasion and levels of LOX expression were examined by Transwell and qPCR assays. (G) Cells were transfected with a Akt siRNA and Akt expression was examined by Western blot. (H\&l) Cells were incubated with NGF for the indicated time intervals or pretreated with PI3K inhibitors then stimulated with NGF; Akt phosphorylation was examined by Western blot. * $p<0.05$ compared with the control group; \# $p$ $<0.05$ compared with the NGF-treated group. 
(A)

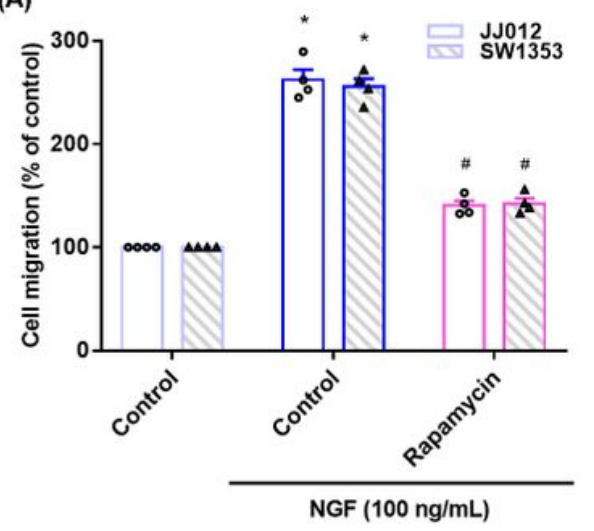

(D)

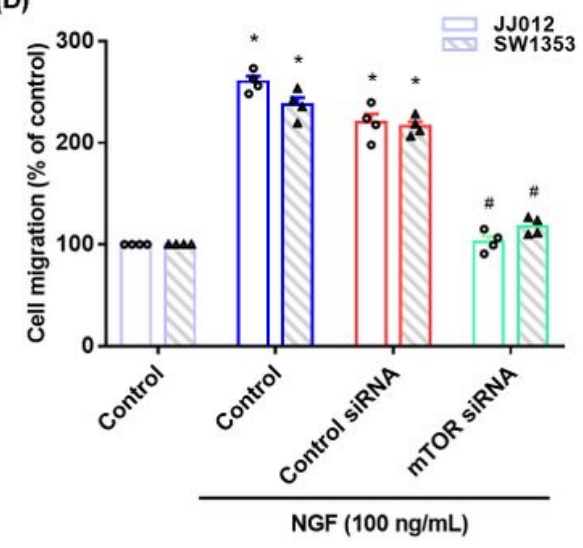

(B)

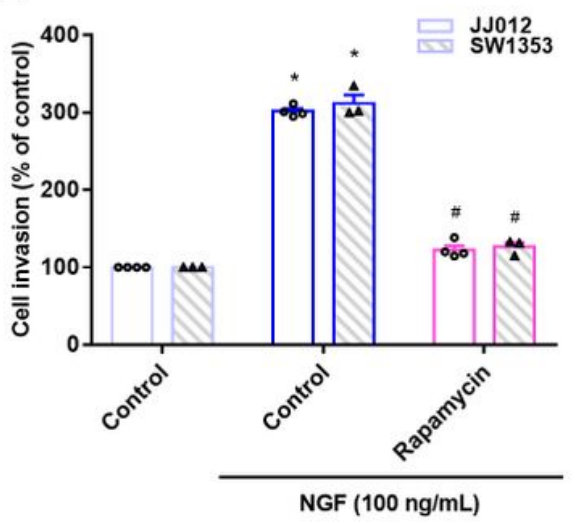

(E)

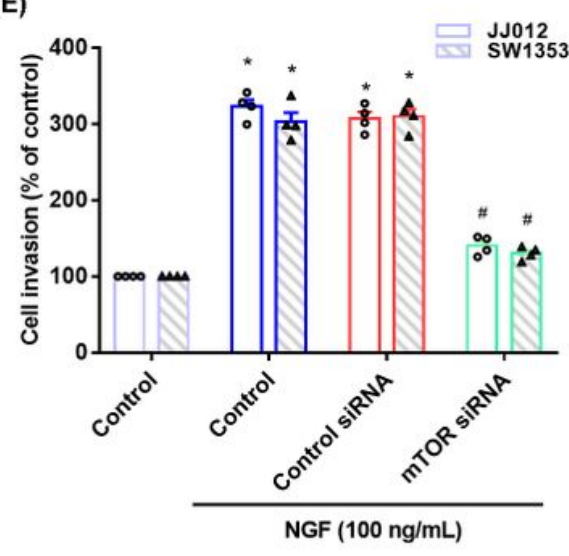

(C)

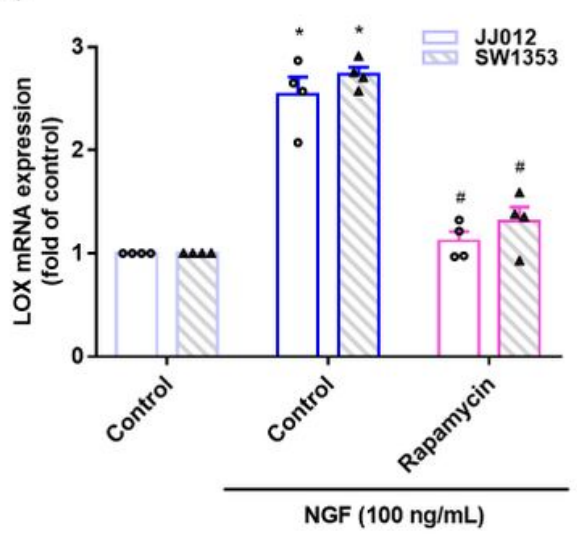

(F)

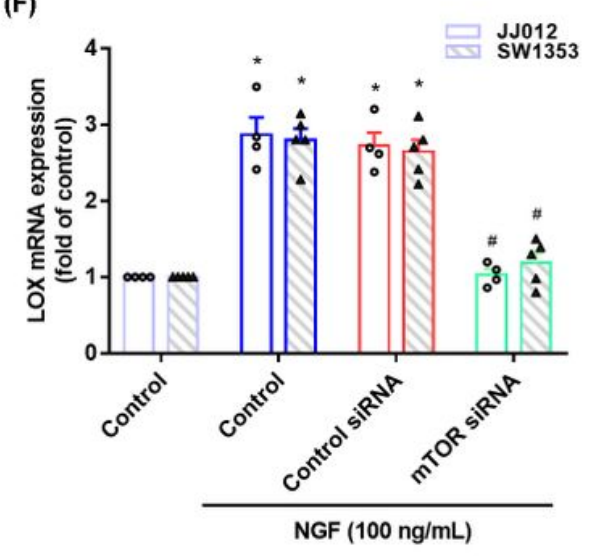

(I)
(G)

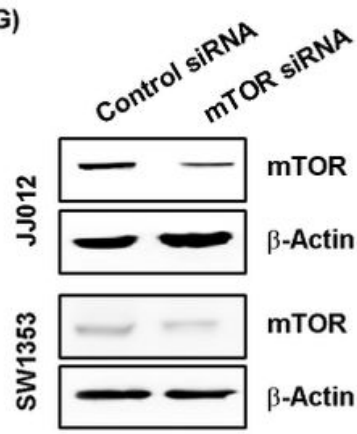

(H)

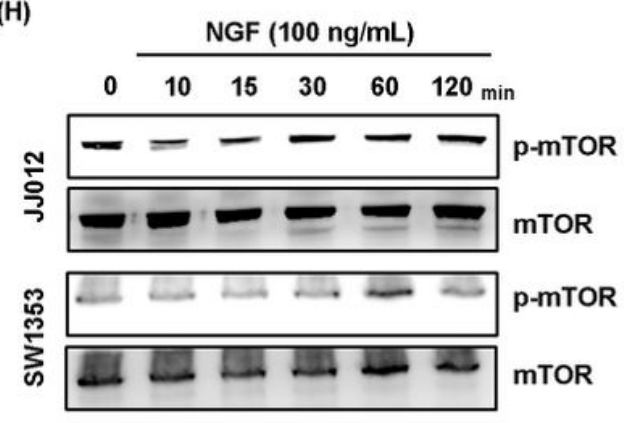

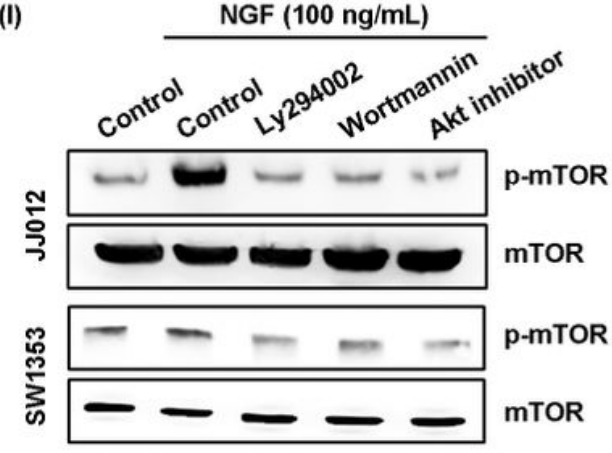

Figure 4

PI3K/Akt-dependent mTOR activation mediates NGF-induced LOX expression and cell motility. (A-F) Cells were pretreated with an mTOR inhibitor (rapamycin) or transfected with an mTOR siRNA, then stimulated with NGF. Cell migratory and invasive activities, as well as levels of LOX expression, were examined by Transwell and qPCR assays. (G) Cells were transfected with an mTOR siRNA and mTOR expression was examined by Western blot. (H\&l) Cells were incubated with NGF for the indicated time intervals or pretreated with $\mathrm{PI} 3 \mathrm{~K}$ and Akt inhibitors, then stimulated with NGF; mTOR phosphorylation was examined by Western blot. ${ }^{*} p<0.05$ compared with the control group; $\# p<0.05$ compared with the NGF-treated group. 
(A)

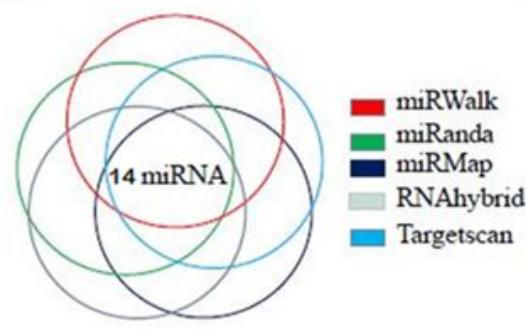

(D)

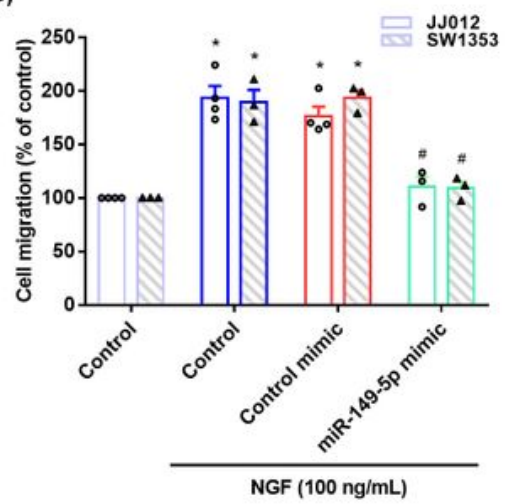

(G)

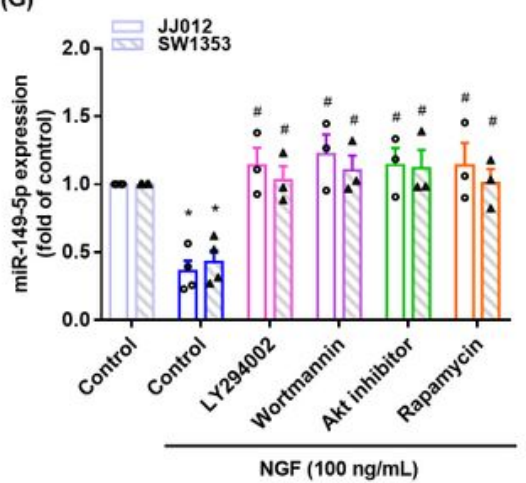

(J)

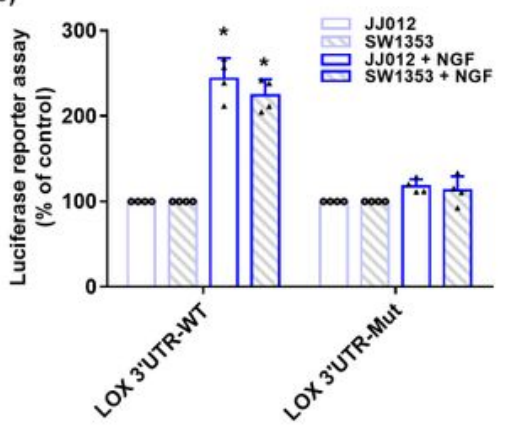

(B)

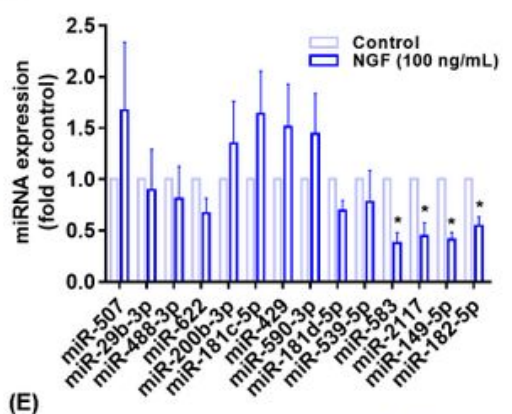

${ }^{400}+* \quad \square \quad$ JJ012

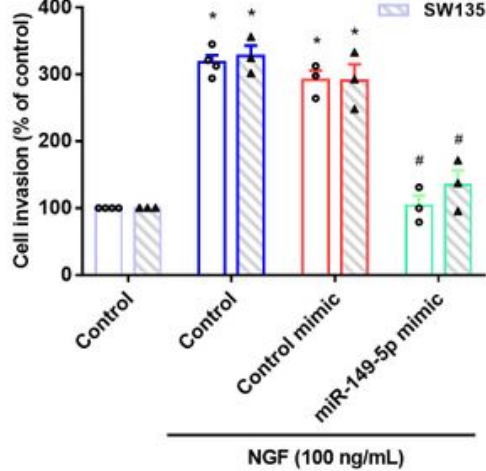

(H)

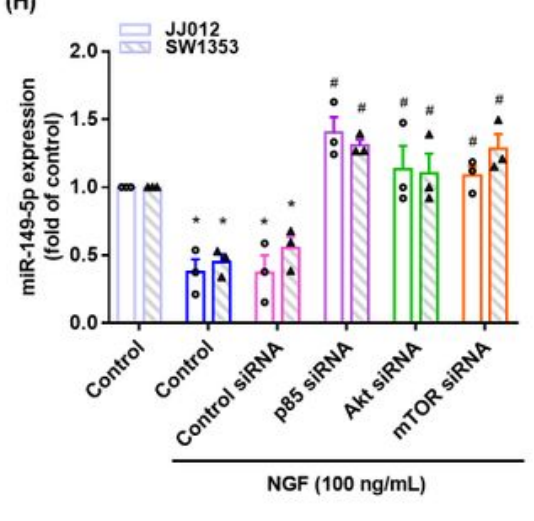

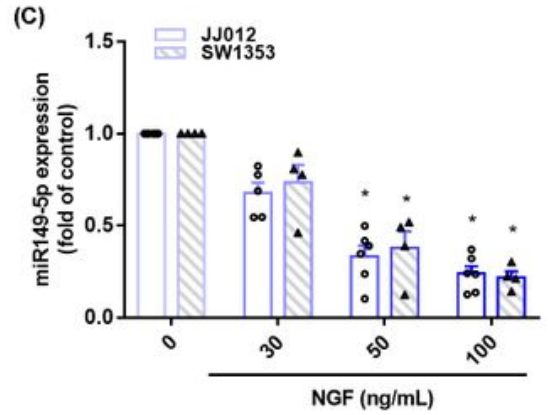

(F)

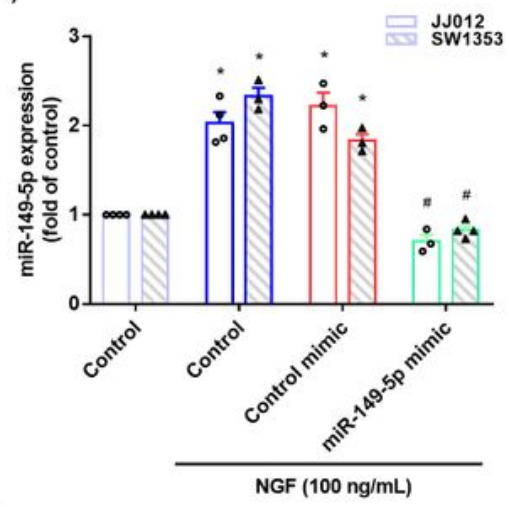

(I)

miR-149-5p binding site

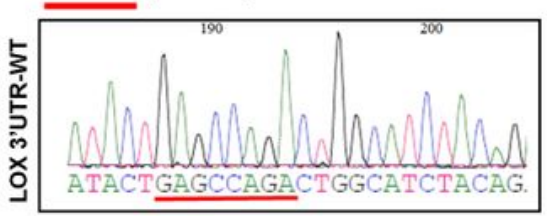

Mutation site

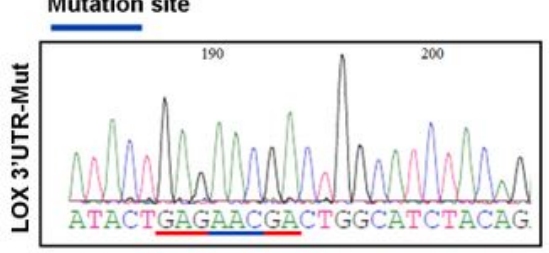

\section{Figure 5}

Inhibition of miR-149-5p mediates NGF-induced promotion of LOX expression and motility of human chondrosarcoma cells. (A\&B) MiRNA target prediction software was used to identify miRNAs that potentially bind to the LOX 3'-UTR. (C) SW1353 and JJ012 cells were incubated with NGF and miR-149-5p levels were examined by qPCR. (D-F) Cells were transfected with miR-423-5p mimic, then stimulated with NGF. Cell migratory and invasive activities, as well as levels of LOX expression, were examined by 
Transwell and qPCR. (G\&H) Cells were pretreated with PI3K, Akt and mTOR inhibitors, or an siRNA, then stimulated with NGF prior to qPCR analysis of miR-149-5p levels. (I) The wild-type and mutant LOX 3'UTRs contain the miR-149-5p binding site. $(\mathrm{J})$ Cells were transfected with 3'-UTR plasmids as indicated, then stimulated with NGF. Luciferase activity was examined. * $p<0.05$ compared with the control group; $\# p<0.05$ compared with the NGF-treated group.

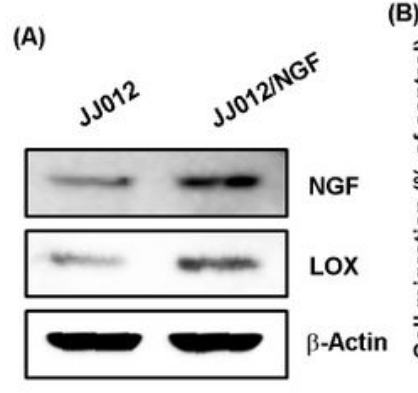

(D)

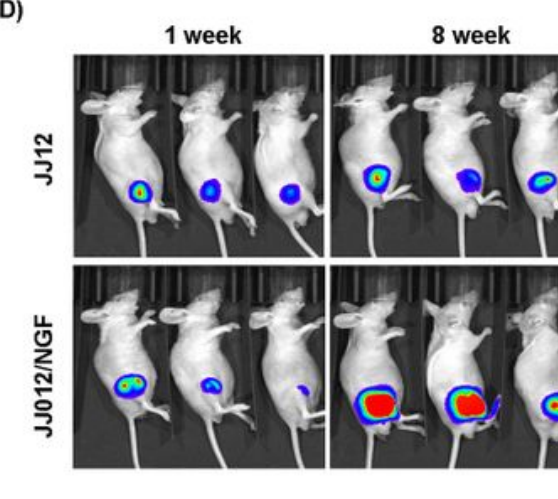

(F)

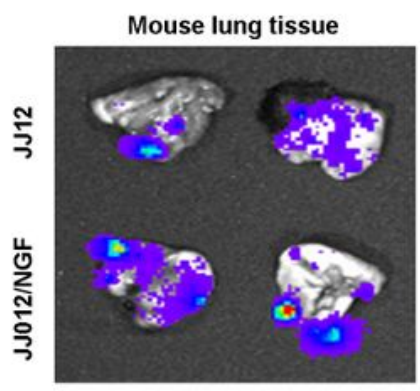

(G)

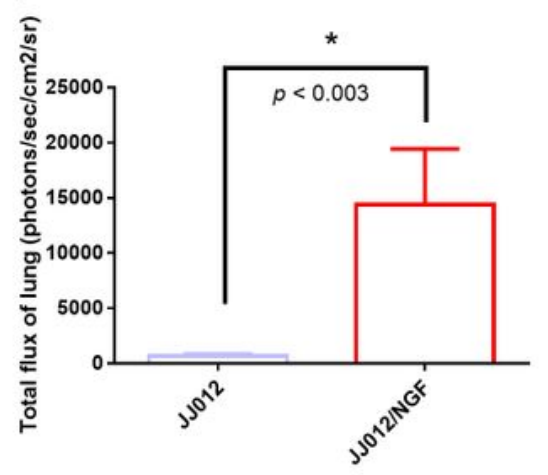

(C)
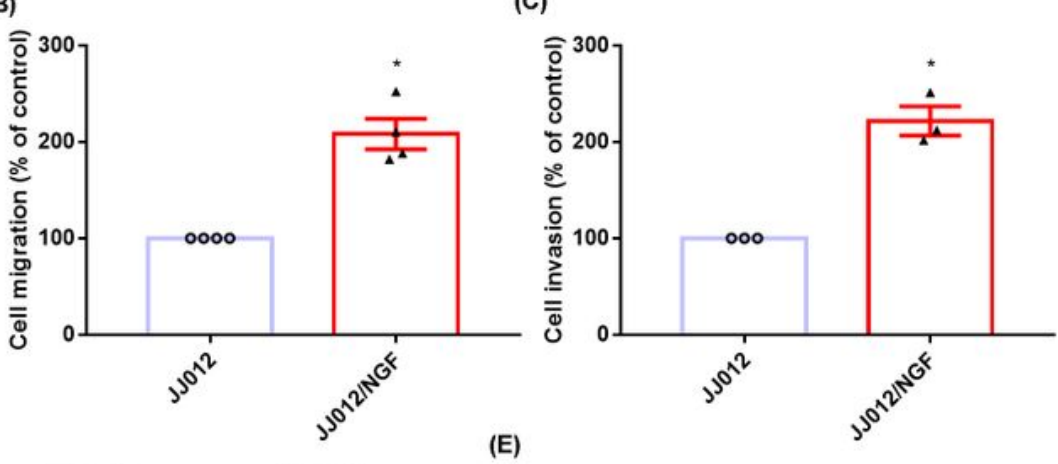

(E)
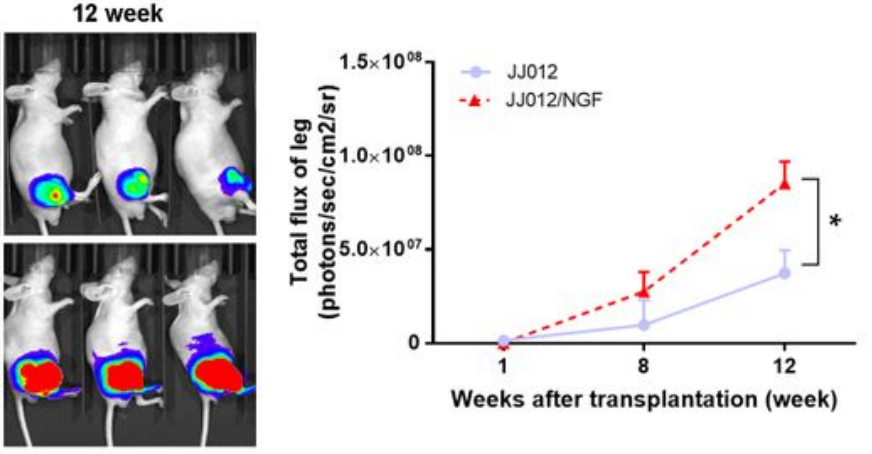

(H)
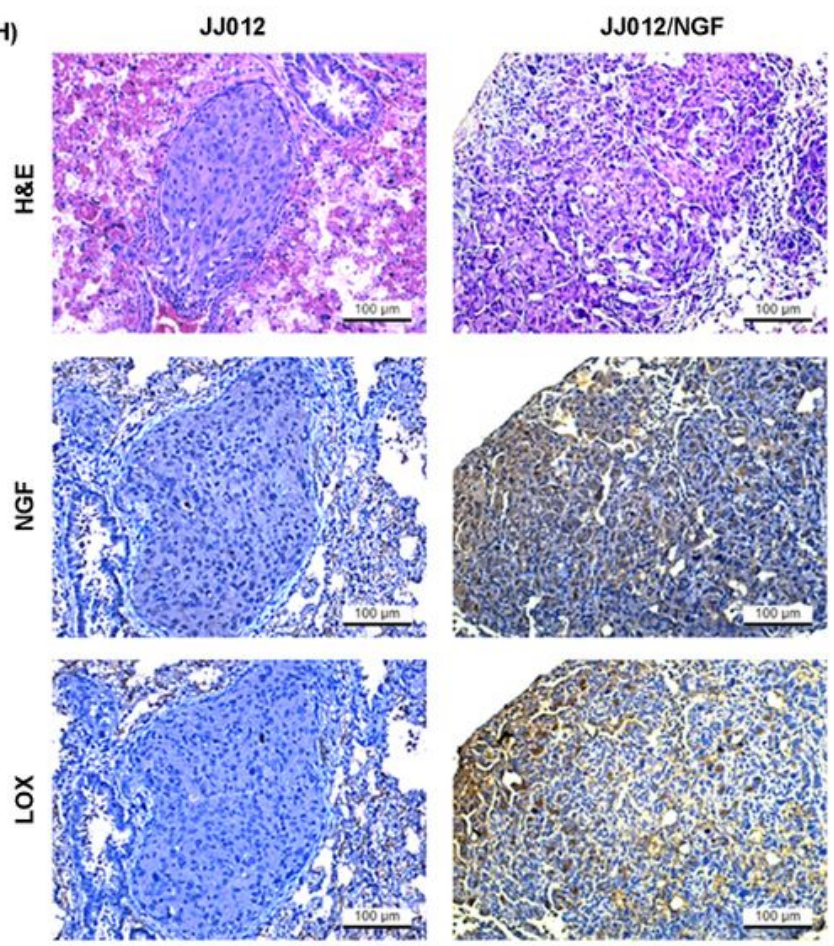

Figure 6 
NGF promotes chondrosarcoma metastasis to lungs in vivo. (A-C) NGF and LOX levels, as well as the migratory ability of JJ012 and JJ012/NGF cells, were examined by Western blot and Transwell. (D-E) The mice were injected with JJ012 or JJ012/NGF cells. Lung metastasis was monitored by bioluminescence imaging at the indicated time intervals, then quantified by photon images. (F-G) After 12 weeks, the mice were humanely sacrificed and the lung tissue was excised, photographed and quantified. $(\mathrm{H})$ Levels of NGF and LOX expression in lung tumors were subjected to IHC analysis. ${ }^{*}<0.05$ compared with the JJ012 group.

(A)

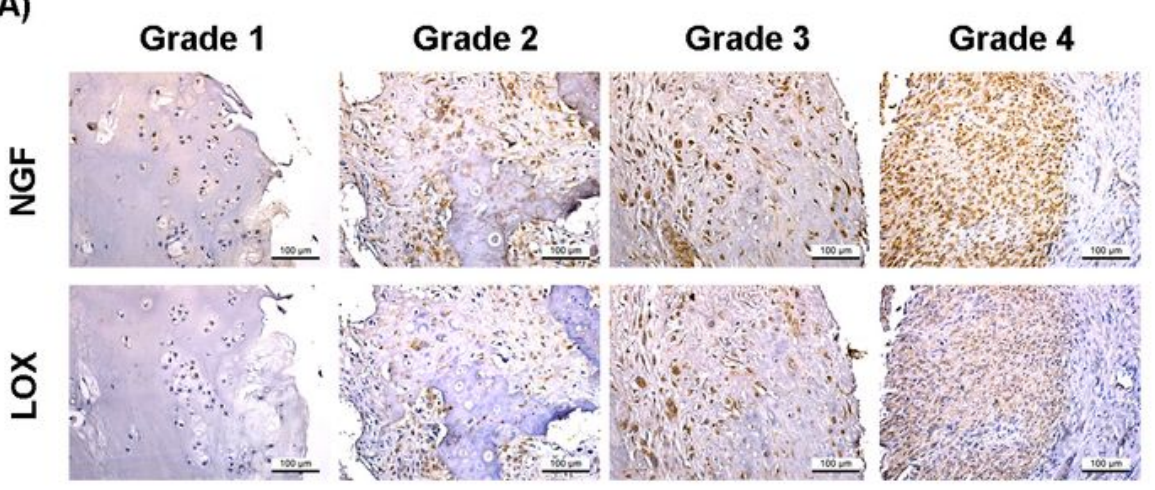

(B)

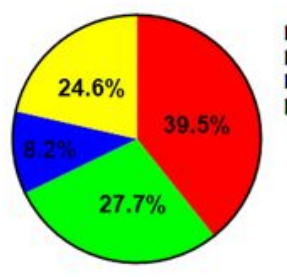

(C)

(D)
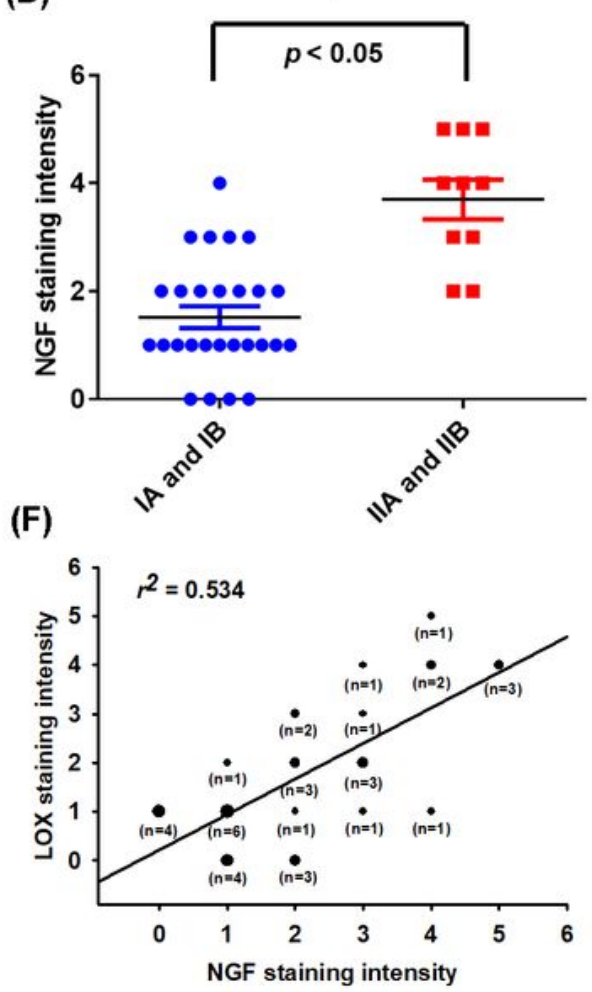

(E)

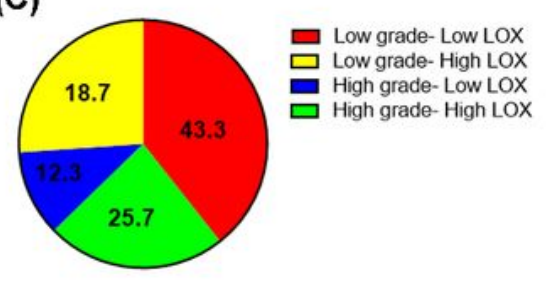

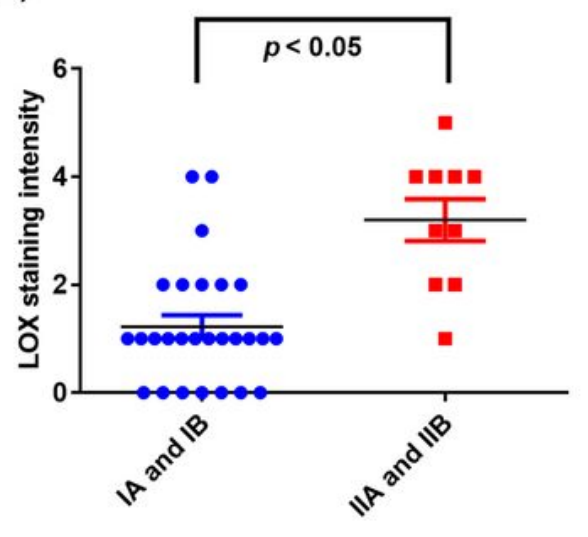


Figure 7

NGF and LOX levels correlated with chondrosarcoma tumor stage. (A-E) IHC stained tissue samples from chondrosarcoma patients were stained with NGF and LOX antibodies, then photographed and quantified. (F) Levels of NGF and LOX were positively correlated. ${ }^{*} p<0.05$ compared with the early-stage (IA and IB) tumor group.

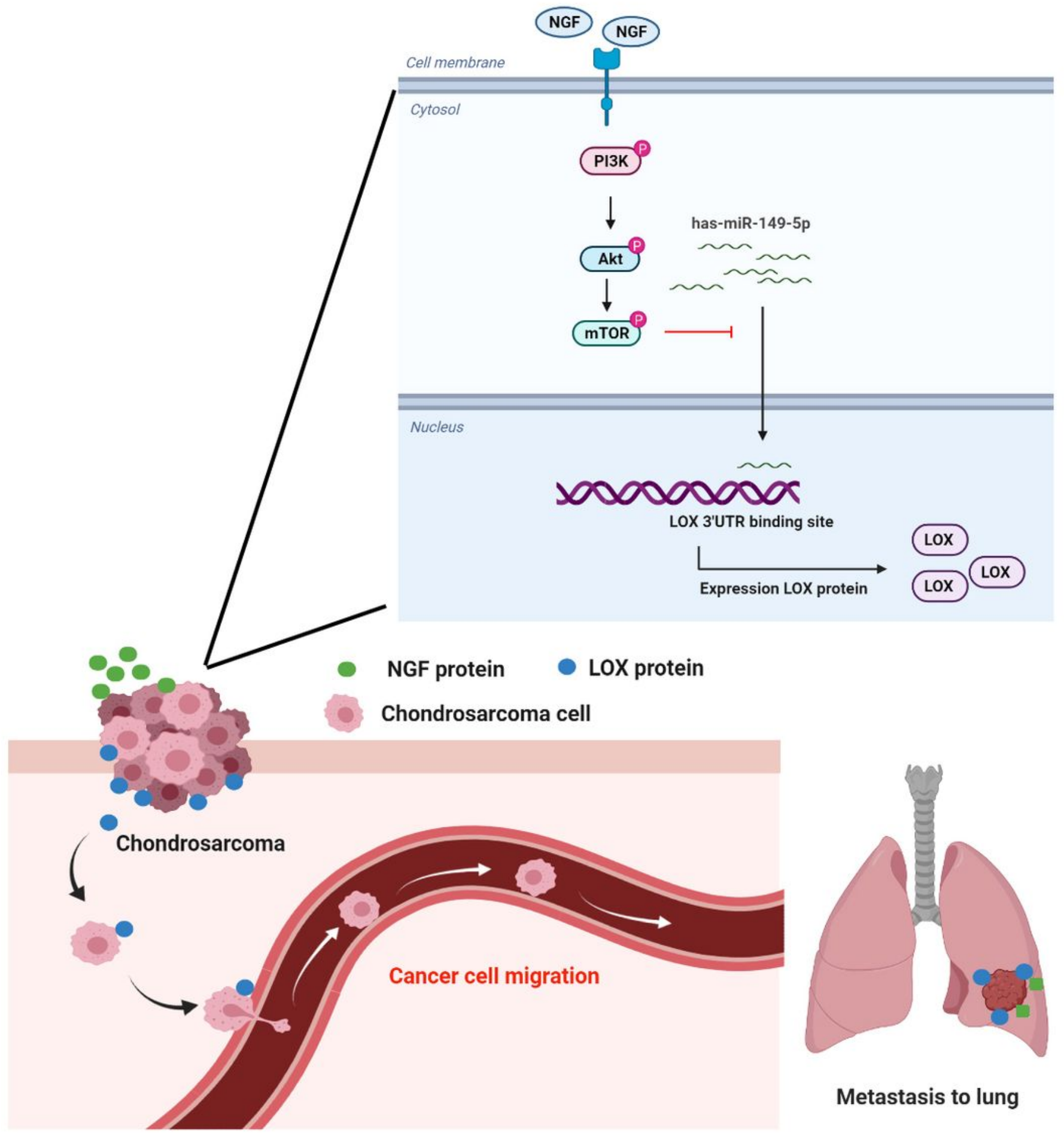

Figure 8 
Schema illustrating the effects of NGF in chondrosarcoma metastasis. NGF promotes LOX-dependent metastasis of chondrosarcoma by suppressing miR-149-5p synthesis via the PI3K, Akt and mTOR signaling pathways. 